\title{
PERIOD CHANGES AS EVIDENCE FOR EVOLUTION IN $\omega$ CEN
}

\author{
EMILIA PISANI BELSERENE \\ Dept. of Astronomy, Columbia University; Dept. of Physics and Astronomy, \\ Lehman College of the City University of New York, N.Y., U.S.A.
}

Much has been said at this colloquium to the effect that observed changes in the periods of variable stars are so fast or so abrupt or so quick to change sign that they cannot possibly be attributed to stellar evolution. If you are wondering how this report can dare to make a contradictory claim read the title carefully. It does not promise that there really is evidence for evolution in Omega Centauri. It only proposes to look at the period changes with evolution in mind. We may all decide that the evidence is strong or that it is weak or perhaps that there is no evidence at all.

The stars in this study are the 43 most regular and best observed of the $\mathbf{R R}_{a b}$ variables in the cluster (NGC 5139). As usual the test of the correctness or regularity of a period begins with the deviations, $O-C$, of observed times from predictions based on an assumed constant period. If the $O-C$ diagrams were reproduced here you would see that for some of the stars a parabola, i. e., a constant rate of change of period, is as good a representation of the observations as one could expect. You would also see several cases where the behavior is clearly too complex to be satisfied by so simple a curve as a parabola and others where a straightline, implying no change in period, is entirely satisfactory. But being able to get away with a constant period does not mean that the period has really been constant nor does the presence of clearly nonevolutionary behavior mean that the effect of evolution is necessarily absent. It is good to have an objectively assigned set of numbers among which to look for evidence of evolution, even though we recognize that the period may not have changed at all or, if it has, that a secular change is certainly not the whole story and perhaps is not even part of it. We can derive from the observations some sort of average, apparent rate of change. It is always possible to force a parabola through any sort of $O-C$ plot, after all, by the simple expedient of combining the epochs into three mean points.

This is what I have done and have found, as Martin (1938) already found in his extensive study of Omega Cen, that the rate of change of period - let us call it $\beta$ as Martin did but decide to measure it in cycles per million years - is positive for some stars and negative for others but that positive values predominate. Before we look at the results in detail let us consider various processes which can affect the observed rate of change of period.

Including stellar evolution there are at least six reasons to expect observations at three epochs to show a deviation from a perfectly constant period. The one that comes to mind first is random observational error. The mean error of the magnitudes is typically about $0.05 \mathrm{mag}$. This leads to a mean error in the epochs of about $10 \mathrm{~min}$, depending on the number of observations. The error in a mean period goes as the 
reciprocal of the time interval and the error in a value of $\beta$ calculated from three epochs, i.e., two time intervals, goes as the inverse square, turning out to be of the order of one-tenth of a cycle per million years.

Next there may be some sort of cosmic mean error, caused by failure of the light curves to repeat exactly. They may actually be multiply periodic, as in the Blazhko effect, but they look irregular if the observations are at more or less random intervals. The effect on the light curves is mostly just scatter, very much like the effect of observational error. Stars with obviously irregular light curves have not been included in this study, but minor irregularities almost certainly occur and we can expect them to cause fluctuations of several minutes in the epochs and again errors of something like 0.1 cycle in $\beta$.

A third way to get a non-zero value of $\beta$ is a different sort of random process. Is it possible that in the pulsation of a star the lengths of individual cycles deviate just slightly and randomly from a basic period (Sterne, 1934)? Fluctuations in period tend to accumulate, whereas fluctuations in epoch tend to cancel out. The effect on an average period goes as the inverse square root of the time interval instead of as the reciprocal. The effect on $\beta$ goes as $t^{-1.5}$ instead of as $t^{-2}$. Individual cycles would only need to differ from the basic pulsation period by a few tenths of a minute to produce an observable effect on $\beta$. Can the theoreticians envisage this much disorder in the pulsation, perhaps through some interaction between pulsation and convection? Can any of us intuitively expect a fluid star to be more orderly than this in its pulsation?

A fourth possibility is another sort of cosmic noise: jumps in period which occur not at each cycle but separated by intervals of weeks, months or years during which the period is relatively stable. Abrupt changes are not attractive from a theoretical point of view, but this sort of behavior is strongly suggested by the many $\mathrm{O}-\mathrm{C}$ diagrams that look like several connected line segments, as Prof. Kukarkin reminded us in his review paper.

These four processes, which can be lumped together under the term random effects, are equally likely to cause negative as positive values of $\beta$. A fifth possibility to bear in mind is that the observations could be affected by some sort of error that has a systematic rather than a random effect on the calculated values of $\beta$.

Finally secular changes in the sizes of the stars are a sixth possibility for producing a $\beta \neq 0$, through the dependence of pulsation period on mean density.

The observational results are presented in summary form in Figure 1 as histograms showing the frequency distribution of the observed values of $\beta$, defined as $(1 / P)$ $(\mathrm{d} P / \mathrm{d} t)$ and expressed in cycles per million years. Look first at the lower figure. The mean epochs are 1893-98 (Bailey, 1902), 1931-35 (Martin, 1938) and 1963-67, the latter based on unpublished magnitudes from the Yale-Columbia Southern Station. A conspicuous feature of the distribution is its broad wings. Several of the rates are quite rapid, $+2.5,-1.1$, and -1.7 cycles per million years. Several more are between a half-cycle and one cycle in absolute value and there is one extreme case: V 104 is decreasing its period at the spectacular rate of 23 cycles per million years. Surely something besides evolution is going on here. Notice next the narrow core of the 


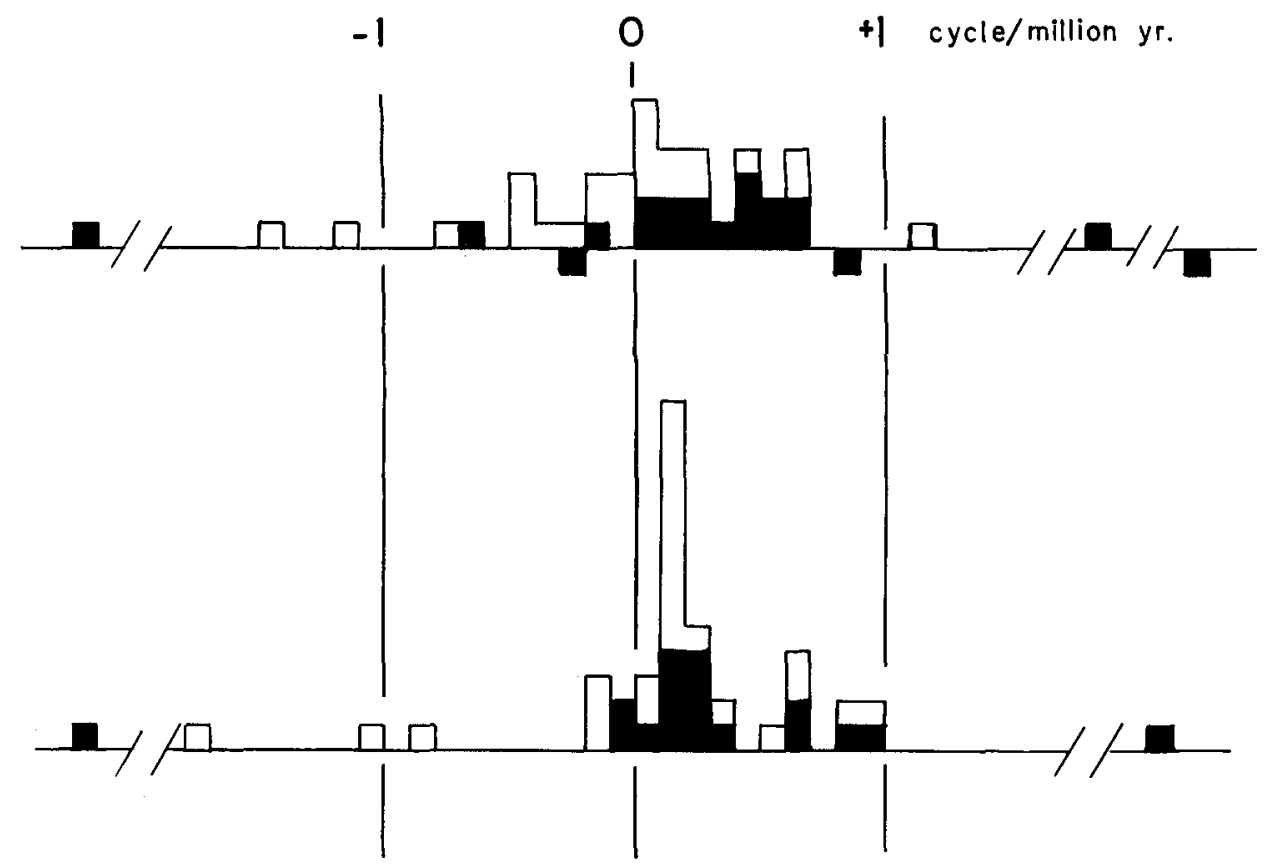

Fig. 1. Distribution of period changes observed in Omega Centauri. Abscissa: $\beta \equiv(1 / P) /(\mathrm{d} P / \mathrm{d} t)$; ordinate: number of stars. Epochs for the lower histogram are 1893-98, 1931-35, 1963-67; epochs for the upper are 1931-35, 1947-48, 1963-67. The shaded portion is the part due to stars with periods longer than two-thirds of a day. The three boxes below the line in the upper figure are for stars that had not been observed in 1893-98.

distribution. About half of the stars seem to form a sub-group with slowly increasing periods, in the vicinity of two-tenths of a cycle per million years. Is evidence for evolution being heard above the noise, or can we account for the positive bias through systematic error and/or, by way of sampling error, through one or more of the random processes?

The median value of $\beta$ is +0.18 cycle per million years. The mean is a less significant statistic because it is much more sensitive to the extreme values, which are very extreme indeed. Arbitrarily excluding V 104 and the two next most extreme on each side we find $\langle\beta\rangle=+0.24 \pm 0.05$ cycle. The dispersion of the 38 values around this mean is 0.32 . Very little of the dispersion can be attributed to mean error of observation or fluctuation in epoch. The width of the central core may be due to these processes, but not the broad wings. The really large positive and negative values of $\beta$ require a different explanation. Not evolution because the time scales are too short. If stars evolve so rapidly through the RR Lyrae stage they will be too uncommon in the population to contribute to our sample. We are almost certainly seeing some sort of average over the third and/or fourth of the random processes considered above, namely the accumulation of small cycle-to-cycle differences or larger less frequent abrupt changes. 
Having decided that random processes are at work in producing some features of the observed distribution let us see whether, through sampling error, they can account for the positive bias. Prof. Kukarkin, in his review paper, asked for the application of modern mathematical statistics. I have applied only a simple Chi-square test, testing the hypothesis that the observed number of positive and negative values of $\beta$ came about through chance selection of 43 cases from a population with equal numbers of positive and negative values. The observed numbers are 34 and 9 , respectively. The probability is less than $5 \%$ that this much difference came about through such chance selection. A statistician would call this a significant deviation from the hypothesis tested and would ask us to reject the idea that positive and negative numbers are equally common in the parent population from which we have drawn our sample.

Statisticians and astronomers have different meanings for the word population. The result of the Chi-square test is not the same as denying that positive and negative period changes occur with equal frequency in a population consisting of all possible RR Lyr stars like these in Omega Cen. Are our numbers affected by systematic error?

Martin (1938) already applied whatever tests he could to his data to try to account for the positive bias by systematic observational error. His first epoch was Bailey's, the same one that I have used, but his second and third were his 1931 and 1935 observations considered separately. He redetermined the phase shifts between his 1931 and 1935 observations by a method that would eliminate the effect of any change in the magnitude system in the meantime. The result was a slight (but not significant) increase in the mean value of the rate of change of period. Then, because the first epoch was based on longer exposures, he considered whether the older observations might require a correction to account for distortion of the light curve. The required correction came out to be longer than the whole exposure time. We are now able to leave Bailey's observations out entirely if we still suspect that something might be wrong there. The upper portion of Figure 1 shows the result of calculating $\beta$ from twentieth century observations only, using Yale-Columbia observations from 1947-48 as a mean point between the 1931-35 and the 1963-67 epochs. The dispersion is greater, as is to be expected, because the random processes have more influence on $\beta$ when the time interval is shorter. The positive bias is still present, which shows that it had not been caused by some sort of systematic error in the 19th century observations. And yet something must be systematically wrong. The median is only +0.10 cycle per million years compared with +0.18 . The median of Martin's values for these 43 stars was +0.30 . Table I shows these along with other statistics of the three distributions. The $\beta$ 's increase, on the average, as we go from the latest back to the earliest selection of epochs. The differences seem a little too large to be merely statistical fluctuations. We are not going to suspect that stellar evolution was faster in the first third of the century than in the second! Apparently some unspecified systematic error has been at work to cause the differences. It can not, however, be the cause of the persistent positive bias. Table II shows the correction 
TABLE I

Parameters of the distribution of $\beta$

\begin{tabular}{lllll}
\hline Epochs & $\begin{array}{l}\text { Median } \\
(N=43)\end{array}$ & $\begin{array}{l}\text { Mean } \\
(N=38)\end{array}$ & $\begin{array}{l}\text { Dispersion } \\
(N=38)\end{array}$ & $\begin{array}{l}\text { Obs. error } \\
(\mathrm{m} . \mathrm{e})\end{array}$ \\
\hline $\begin{array}{llll}1931-35 \\
1947-48\end{array}$ & +0.10 & $+0.11 \pm 0.06$ & 0.36 & 0.08 \\
$1963-67$ & & & & \\
$1893-98$ & & $+0.24 \pm 0.05$ & 0.32 & 0.05 \\
$1931-35$ & +0.18 & & & \\
$1963-67$ & & & & 0.16 \\
$1893-98$ & & & & \\
1931 & +0.30 & $+0.33 \pm 0.07$ & 0.41 & \\
1935 & & & & \\
\hline
\end{tabular}

TABLE II

Hypothetical corrections, which, if applied to the midpoints of the exposures, would reduce the derived values of $\beta$ by 0.1 cycle per million years

\begin{tabular}{llcccc}
\hline & $1893-98$ & 1931 & 1935 & $1947-48$ & $1963-67$ \\
\hline & & \multicolumn{1}{c}{$-16^{\mathrm{m}}$} & $+7^{\mathrm{m}}$ & $-13^{\mathrm{m}}$ \\
& $-66^{\mathrm{m}}$ & ${ }^{+32^{\mathrm{m}}}$ & & $-61^{\mathrm{m}}$ \\
ET-UT & $-0^{\mathrm{m}} \cdot 1$ & $+3^{\mathrm{m}}$ & $-4^{\mathrm{m}}$ & & \\
Exposure & $30^{\mathrm{m}}$ & $+0^{\mathrm{m}} 4$ & $+0{ }^{\mathrm{m}} 4$ & $+0{ }^{\mathrm{m} 5}$ & $+0{ }^{\mathrm{m}} 6$ \\
\hline
\end{tabular}

to each epoch in each group of three that is required to reduce the resulting values of $\beta$ by about 0.1 cycle per million years. Also listed are the values of ET-UT, which are negligible and have not been applied, and the lengths of typical exposures. Only the most artificial and unreasonable selection of 'corrections' would reduce the $\beta$ 's by the required amounts.

The observed values of $\beta$, then, are the results of random observational error, cosmic fluctuations in epoch and/or period, systematic error of unknown origin, and something else, something that can account for an average real increase in period at the rate of some two-tenths of a cycle per million years. The evidence for evolution in Omega Centauri is that secular increase in the sizes of the stars is a reasonable way to provide the increase.

Iben and Rood (1970) have shown that low- $Z$ models with appropriate parameters of age, etc., arrive on the horizontal branch to the blue of the instability strip and cross it evolving to the red with periods increasing at the rate of a few tenths of a cycle per million years, which is in agreement with the observations in Omega Cen provided that we attribute the negative observed values to larger, non-evolutionary 
fluctuations superimposed on the secular changes. If their theory is correct there is a range in mass, with the lower-mass stars crossing the strip more rapidly and at higher luminosities. The observations do not show a correlation of $\beta$ with magnitude but perhaps this is only because magnitudes of variable stars in a crowded field are not precise enough. The mass range should also produce a correlation of $\beta$ with period since the less massive, more luminous stars are less dense as they cross the strip and therefore of longer period. This correlation has been observed (Martin, 1938; Belserene, 1964); the shaded portion of the histograms in Figure 1 is the part due to stars with periods longer than two-thirds of a day. This portion does indeed favor increasing periods more strongly than the rest. A roundabout way of looking for a relation between $\beta$ and luminosity is with the help of a period-amplitude relation such as Figure 2, where the kind and size of symbol shows the sign and size of $\beta$. The positive values of $\beta$ and particularly the larger ones tend to occur not only at the longer periods but especially where the period is long for the amplitude. We can interpret this as the correlation of $\beta$ with magnitude if we simply look at the diagram from another angle. Tilt the page counterclockwise until the lines of constant period

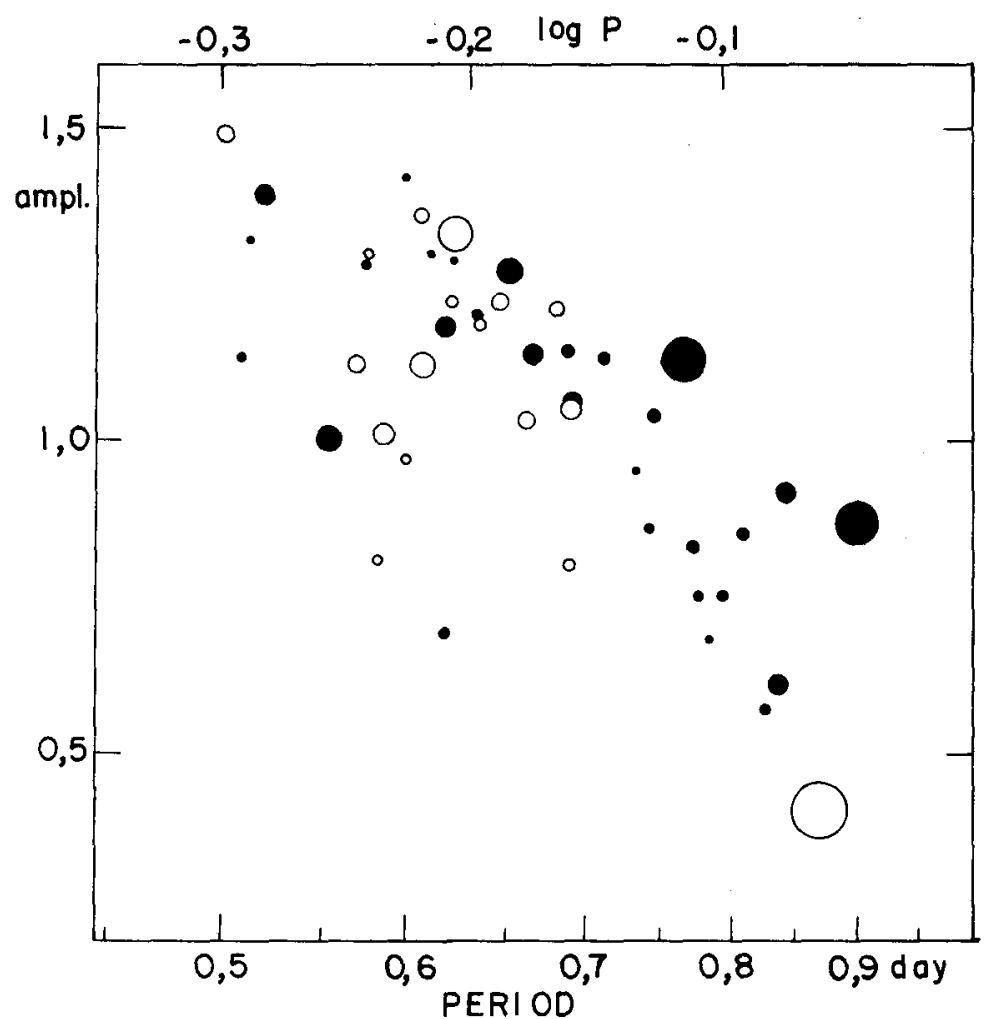

Fig. 2. Period-amplitude relation for 46 regular $\mathbf{R R}_{a b}$ variables in Omega Centauri. Filled circles: increasing periods; open circles: decreasing periods; larger dots mean faster rates. The rates are from the epochs: $1931-35,1947-48,1963-67$. 
are more nearly horizontal than vertical. This is rather like the way the lines of constant density cross the horizontal branch. The lines of constant amplitude now slant steeply up to the right, and the line of zero amplitude has become the red edge of the instability strip. Viewed in this way the period-amplitude plane has become a somewhat distorted version of a piece of the color-magnitude plane. The stars have entered the $\mathbf{R R}_{a b}$ region on their way to the right from a blue horizontal branch and the more luminous ones are going fastest. There are many exceptions in Figure 2, but we account for most of those by the superposition of random on secular processes, especially at the shorter periods near the transition from $R_{R_{c}}$.

Such is the evidence for evolution in Omega Cen: present but not very strong. This is not to say that evolution has been at work whenever there is curvature in an $O-C$ diagram. In fact, when the curvature is obvious the rate is usually too fast to be secular. To say that evolution has been detected in Omega Cen is not the same as claiming that it accounts for all period changes observed in this and other clusters or even that it can ever be detected in other clusters. Omega Centauri is very unusual, both variable-rich and variable-poor, rich in number of variable stars but poor when this number is normalized to account for the total population of the cluster (Belserene, 1956; Kukarkin, this colloquium). Only a variable-rich cluster will have a large enough sample of stars to show a trend in the presence of noise. Even then the rate of evolution will be orders of magnitude slower than the noise unless the stars pass through the RR Lyrae phase rather rapidly. If they do, the cluster is necessarily poor in variables in proportion to the total number of stars. Omega Centauri is a very favorable case.

\section{References}

Bailey, S. I.: 1902, Ann. Harvard Coll. Obs. 38.

Belserene, E. P.: 1956, Contr. Rutherfurd Obs. No. 33, 13.

Belserene, E. P.: 1964, Astron. J. 69, 475.

Iben, I., Jr. and Rood, R. T.: 1970, Astrophys. J. 161, 587.

Martin, W. C.: 1938, Ann. Sterrew. Leiden 17, Part 2.

Sterne, T. E. : 1934, Circ. Harvard Coll. Obs. No. 386.

\section{DISCUSSION}

Cox: Do these studies by you and the others include the $\mathrm{RR}_{c}$ variables? Do we have evidence of any $c$-type star changing to an $a b$-type or vice-versa?

Belserene: There were no $\mathbf{R R}_{c}$ 's in this sample. They have larger fluctuations in period and are difficult to follow. We haven't been looking at the ciuster long enough to expect to catch a change in type.

Jones (to Cox): AC And is the only RR Lyrae in the field with a period ratio of roughly $4: 3$ which indicates that the fundamental and first overtone are simultaneously excited. It has no photoelectric light curve. I know of no RR Lyrae which has changed its Bailey type.

Schwarzschild: It seems to me that all the papers we heard this morning agree on one point: Random variations of the period of RR Lyr variables are common, if not the rule - a real challenge to theory. Since these random changes appear percentage-wise to be very small they cannot be observed in any other quantity, which makes the determination of the character of the random period changes extraordinarily important. 
Wesselink: Do stars with Blazhko effect behave differently from the others with respect to period behaviour?

Belserene: The stars known to have a Blazhko effect were not on this program. Unrecognized Blazhko-effect stars probably contribute to the high-dispersion component in the distribution of the period changes.

Aizenman: Is it possible that high order harmonics which may be generated in the atmosphere might interfere with the fundamental mode in a manner that gives rise to a time variation in the maximum of the light curve?

Cox: In all accurate non-linear calculations that $I$ know about, the star pulsates in only a pure mode. An atmospheric phenomenon probably cannot excite a deepseated variation such as these pulsations. 